\title{
Participación de las MIPYMES al desarrollo local, resultado de la actividad turística gastronómica: caso de estudio
}

\section{Abel Efraín Peña-Hernández ${ }^{1}$ \\ Juan Bory Reyes ${ }^{1}$ \\ Francisco Villafaña-Rivera ${ }^{1}$ \\ Cristina Flores-Amador ${ }^{2}$ \\ Josefa Espitia-López ${ }^{3}$}

${ }^{1}$ Instituto Politécnico Nacional

Escuela Superior de Ingeniería Mecánica y Eléctrica Zacatenco,

Sección de Estudios de Posgrado e Investigación

Col. Lindavista, CP 07738, Ciudad de México.

MEXICO.

¿Universidad Autónoma del Estado de México

Cerro de Coatepec, Ciudad Universitaria Toluca,

C.P. 50110, Estado de México.

MEXICO

3Universidad Autónoma del Estado de Hidalgo

Instituto de Ciencias Agropecuarias,

Área Académica de Ciencias Agrícolas y Forestales.

Rancho Universitario. Av. Universidad km 1,

Ex-Hacienda de Aquetzalpa, C.P. 43600, Tulancingo de Bravo, Hidalgo.

MEXICO

correos electrónicos (emails):

phae_55@hotmail.com, juanboryreyes@yahoo.com

frank-31-90@hotmail.com, cristinafloresamador@hotmail.com

josefa_espitia11153@uaeh.edu.mx

Recibido 03-09-2019, aceptado 21-11-2019.

\section{Resumen}

El turismo gastronómico en México es muy diverso, algunos destinos turísticos han adoptado esta iniciativa para atraer un mercado potencial, donde los principales actores del sistema son las micro, pequeñas y medianas empresas (MIPYMES). En el presente trabajo de investigación se estudiaron las relaciones existentes entre los prestadores de servicios y la actividad turística gastronómica en Villa de Tezontepec, Hidalgo, por medio de la Metodología de Sistemas Suaves, que permitió caracterizar la influencia de los actores clave y los elementos internos y externos del sistema correspondiente a las MIPYMES gastronómicas locales, para identificar alcances e impactos reflejados en el desarrollo local.

Palabras clave: sistema, turismo gastronómico, actividad turística, MIPYMES, desarrollo local.

\section{Abstract \\ (Participation of the MIPYMES in local development, result of gastronomic tourism activity: case study)}

Gastronomic tourism in Mexico is very diverse, some tourist destinations have adopted this initiative to attract a potential market, where the main actors of the system are micro, small and medium enterprises (MSMEs). In the present research work, the relationships between the service providers and the gastronomic tourism activity were studied in Villa de Tezontepec, Hidalgo, through the Soft Systems Methodology that allowed to characterize the influence of the key actors, internal and external elements. of the corresponding system to the local gastronomic MIPYMES and thus identify the scopes and impacts reflected in the local development.

Index terms: system, gastronomic tourism, tourist activity, MSMEs, local development.

\section{Introducción}

El turismo es un fenómeno social, cultural y económico que activa el desarrollo local a través del comercio, bares, restaurantes, hospedaje, agencias de turismo, artesanías, entre otros; promueve acciones desde el poder público además de que produce ingresos y empleo. En México es considerado una fuente de desarrollo que genera impactos dentro de la sociedad receptora; es uno de los sectores económicos más importantes y dinámicos en el mundo actual, tanto por su nivel de inversión y aportación de divisas, como por su contribución al desarrollo regional; favorece directamente el 9\% del PIB mundial y genera uno de cada once empleos [1], [2], [3], [4].

México está experimentando un auge en el turismo internacional; 2015 fue un año récord con 32.1 millones de turistas internacionales que contribuyeron con 246.1 mil millones de 
pesos mexicanos a la economía. En un periodo que abarca 2014 hasta el segundo semestre del 2017 el ingreso de divisas por concepto de viajeros internacionales fue de 14706 millones de dólares. El aumento del flujo monetario hacia México, producto del aumento del turismo ha superado el crecimiento en muchas economías turísticas avanzadas y emergentes. En este sentido, los gobiernos contemplan el turismo como un área para el desarrollo y la aplicación de políticas [5].

De acuerdo con Varisco [6] y el Instituto Latinoamericano y del Caribe de Planificación Económica y Social - ILPES [7], el turismo puede considerarse desde tres perspectivas: la diversidad de actores que intervienen; la complejidad de los impactos que el turismo produce en la sociedad; los diferentes modelos de desarrollo turístico. Por un lado, los emprendedores locales utilizan el potencial que tienen a su alcance para conseguir que sus productos sean competitivos; por otro, las instituciones y los valores socioculturales locales se convierten en la base de los procesos de desarrollo.

En esta visión sistémica surge la idea de territorio, que se entiende como un espacio construido socialmente y definido por sus actores y las relaciones entre ellos. Es capaz de reflejar con detalle la senda de desarrollo como resultado de las decisiones contextuales de sus actores. De acuerdo con lo anterior, la sostenibilidad del desarrollo turístico remite necesariamente a la elaboración de metodologías integradoras, con un enlace más amplio que las técnicas de gestión ambiental [8], [9].

En el área de los instrumentos del desarrollo las iniciativas locales contribuyen a la búsqueda de un camino que conduce a la salida de la crisis; su fortaleza reside en los instrumentos que favorecen un aumento de la productividad que no reside en los bienes y servicios, sino en las personas, donde la realización personal y colectiva se prioriza sobre la función del consumo [10], [11], [12], [13].

Desde una perspectiva de sistemas, el turismo es un factor principal de desarrollo que se centra en el aprovechamiento de los recursos, pues ofrece un panorama estable que mejora la calidad de vida de la sociedad como una cadena, donde el desarrollo local envuelve diversos actores que generan un crecimiento más allá de lo interno. El diseño de un sistema de desarrollo local basado en el turismo gastronómico es una manera de demostrar los alcances generados y el aprovechamiento adecuado de los recursos bajo el establecimiento del sistema como principal factor de crecimiento derivado de la actividad turística. De este modo, el turismo gastronómico es un detonante del desarrollo, y ha surgido como una tendencia en crecimiento donde los visitantes se desplazan con la finalidad de conocer y degustar los platillos típicos de la lo- calidad que desean conocer. Se trata de un sistema con múltiples relaciones de crecimiento en la comunidad emisora.

\section{Desarrollo}

Algunas investigaciones demuestran que el desarrollo económico local depende de la capacidad de integrar el aprovechamiento sostenible de los recursos, al movilizarlos hacia la satisfacción de las necesidades y los problemas básicos de la población local [14], [15]. Los actores sociales principales para el desarrollo local son empresas y emprendedores, asociaciones empresariales y profesionales, gobierno, administración pública, ONG, universidades y centros de investigación. Una estrategia en donde los actores son los beneficiarios, las aspiraciones y prioridades en materia de desarrollo crean problemáticas singulares; se requiere que los actores públicos y privados ejecuten programas de inversión de manera coordinada con iniciativas en las que los proyectos económicos y sociales se coordinan y gestionan a través de nuevas formas de gobernación [16], [17]. En relación con la manera en que han evolucionado las micro, pequeñas y medianas empresas - MIPYMES, se renuevan en función de las necesidades económicas y sociales, buscando rutas, creando servicios para satisfacer necesidades. Por tanto, debe dejar de considerarse a las MIPYMES como empresas o unidades económicas, en cambio, constituyen organizaciones en las que ocurren procesos y fenómenos; se trata de organizaciones con estructuras, identidades, contextos y procesos decisorios [18], [19].

El turismo representa una función de consumo relacionada con la producción de bienes y servicios que satisfacen las necesidades del visitante. En los sectores de alojamiento, restaurantes y servicios de esparcimiento, culturales y deportivos, integra 433,090 MIPYMES, que representan el 99.8\% de las empresas totales en estos sectores, y generan 1.6 millones de empleos, es decir, el 86.3\% del empleo total en el sector. [20], [21] [22]. Por tanto, se trata del pilar económico fundamental del sector, donde la promoción turística juega un papel importante para mantener y consolidar a México como una potencia mundial, pues tiene el objetivo de generar una mayor demanda para los destinos y atractivos del país [23]. El Instituto Nacional de Estadística y Geografía dio a conocer los resultados de los Indicadores Trimestrales de la Actividad Turística (ITAT) durante el tercer trimestre del 2014: el PIB tuvo un incremento del $3.4 \%$ gracias a la actividad turística. El PIB por concepto del turismo en México está por encima de algunos países de Latinoamérica [24]. las microempresas son una gran fuente generadora de empleo a nivel nacional y regional, tanto en países desarrollados como en países en vías de desarrollo, en el último Censo Económico, las MIPYMES continuaron desempeñando un papel importante dentro de la generación de 
empleos, comparado con el censo del 2009, debido a esto, la mayoría de estas empresas están constituidas por familiares y amigos cercanos, que desempeñan actividades artesanales con la finalidad de prevalezcan las enseñanzas heredadas. Dentro del sector de servicios, las MIPYMES tienen una mayor presencia, que se refleja en el número de establecimientos dedicados a este ramo: del total de establecimientos dedicados a los servicios el $94.4 \%$ son micro, $4.7 \%$ pequeños, $0.5 \%$ medianos, y $0.4 \%$ grandes [25].

Para el desarrollo de MIPYMES turísticas existen grandes oportunidades de inversión ya sea pública o privada. Con la intención de diversificar la demanda de los visitantes, se brindan apoyos para detonar la actividad económica en regiones con dificultades de crecimiento. Gracias a este tipo de apoyos los micro, pequeños y medianos empresarios tienen a su alcance la posibilidad de elevar su producción y mejorar servicios de calidad no solo para el turismo. Durante 2012, SECTUR en coordinación con el Banco Nacional de Comercio Exterior, a través del programa de financiamiento para el sector turismo, apoyó a 116 micro, pequeñas y medianas empresas (MIPYMES) turísticas mediante el otorgamiento de 138.4 millones de pesos, lo que representó incrementos de $33.3 \%$ y de $73.4 \%$ real respecto a lo observado el año anterior [26].

Las MIPYMES son de vital importancia para México con base en el Censo Económico 2009 del INEGI; ese año había en nuestro país 3.7 millones de unidades económicas catalogadas como MIPYMES, las cuales dieron empleo a 13.5 millones de personas, lo que representa $99 \%$ de las unidades económicas del país y $67 \%$ del personal ocupado [27]. Las MIPYMES constituyen la columna vertebral de la economía nacional por los acuerdos comerciales que ha tenido México en los últimos años y por su alto impacto en la generación de empleos y en la producción nacional [28]. En México las PYMES generan 52\% del Producto Interno Bruto (PIB) y $72 \%$ del empleo en el país; el sector turístico se encuentra principalmente representado por la prestación de servicios que requieren el involucramiento de personas y no tan fácilmente pueden ser reemplazadas por la maquinaria, como sí puede hacerse en otros sectores [29].

Respecto del impacto para la economía por parte de las MIPYMES, Nacional Financiera (NAFINSA) facilitará el acceso al financiamiento para las empresas identificadas y referenciadas por SECTUR y Fondo Nacional de Fomento al Turismo (FONATUR), con especial énfasis en las MIPYMES del Sector Turismo; FONATUR proporcionará a estas asesoría para la integración, calificación y seguimiento de proyectos de inversión, de conformidad con las políticas, costos, programas y lineamientos autorizados y aplicables a FONATUR (SECTUR). El turismo de cultura y gastronomía son algunos de los productos turísticos que, en adición y para complementar el producto sol y playa, se están promoviendo con un gran potencial en México [30].

Dentro de este panorama, hay que destacar que la cocina mexicana es la expresión viva del patrimonio, pues combina el tesoro de las tradiciones culinarias con la originalidad de la innovación, lo que la ubica entre una de las más aclamadas del mundo [31]. Como resultado, SECTUR creó las Rutas Gastronómicas de México, de esta forma, nuestro país segmenta la oferta turística para cada tipo de demandantes, con la finalidad de ofrecer una experiencia culinaria a través de los sabores de México, sin olvidar las tradiciones, cultura y atractivos principales generando así una oferta completa [32]. SECTUR dio a conocer que en México el sector gastronómico genera 1.3 millones de empleos directos y 3.3 indirectos. En términos económicos, aporta 1.4\% al PIB nacional y registra un ritmo de crecimiento de casi $4 \%$ anual, por lo que se considera un sector importante [33]. En este sentido, los gobiernos adquieren cada vez mayor conciencia de la importancia del turismo como impulsor económico, a tal grado que lo incorporan para logro de sus políticas y objetivos, en materia de desarrollo [34].

Se debe conocer el perfil del consumidor de turismo gastronómico y ofertar un producto a su medida; para ello es necesario la elaboración de un plan de comercialización que facilite la comunicación de los servicios y productos ofertados; formar y reciclar al personal del sector turístico y crear un sistema de señalización de rutas gastronómicas, bodegas, industrias alimentarias, alojamientos, restaurantes, entre otros [35]. Así, el turismo gastronómico se perfila como una opción viable para el desarrollo de algunas de las etnias que habitan el territorio mexicano [36], pues se presenta al turismo en óptimas condiciones para convertirse en locomotora del desarrollo, gracias a que en las políticas regionales el turismo se ha convertido en casi la única potencialidad, producto de motivación turística y del diseño de líneas estratégicas en las que participan ayuntamientos y particulares [37]. También es posible pensar que el turismo, como actividad socio-productiva, pueda llegar a generarse a partir de acciones endógenas tendientes a producir un desarrollo integral de territorios, en donde hasta el momento no se había pensado como opción desde la comunidad local en su conjunto [38].

\section{Método}

El sistema que ha generado cambios en el municipio Villa de Tezontepec no está determinado. El propósito de este trabajo de investigación es realizar el diseño de un sistema de desarrollo local mediante la integración de las MIPYMES turísticas gastronómicas que influyen en el crecimiento y desarro- 
llo municipal, pues, aunque los cambios en este sentido son visibles, no se ha determinado qué los genera y con qué otros sistemas tienen relación de manera directa y que influyen de forma positiva y negativa.

En el estado de Hidalgo, la actividad económica de servicios se ubica como el primer generador de recursos, de acuerdo con la Secretaria de Desarrollo Económico del Estado de Hidalgo [39]. El Programa Sectorial de la Secretaria de Turismo y Cultura [40] incluye fases de diagnóstico comunitario, evaluación de las variables de desarrollo local y formulación de acciones estratégicas orientadas a propiciar este desarrollo. Basado en el Plan Estatal de Desarrollo 2011-2016, la Secretaria de Turismo y Cultura refrenda el compromiso de fortalecer la profesionalización del sector e impulsar una cultura de calidad, para ofrecer servicios y productos certificados, así como una mayor oferta turística, partiendo del fomento a la inversión y el financiamiento requerido para la utilización del patrimonio natural, histórico y cultural de la entidad.

Villa de Tezontepec se localiza al sur del estado de Hidalgo (véase Fig. 1). Colinda al norte con los municipios de Zempoala

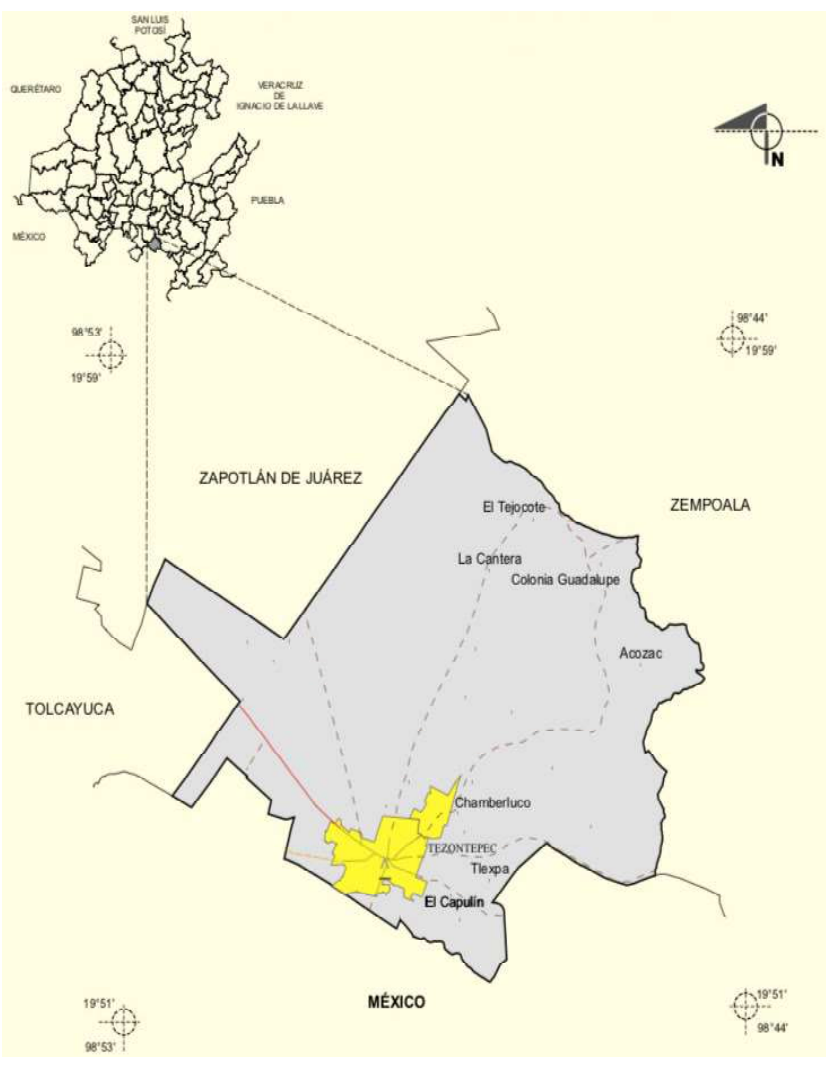

Fig. 1. Focalización del municipio de Villa de Tezontepec, Hidalgo (INEGI, 2009). y Zapotlán de Juárez; al sur con el municipio de Temascalapa, Estado de México; al Oriente con los municipios de Zempoala y Temascalapa, y al poniente con los municipios de Tolcayuca y Zapotlán de Juárez [41]. Su objetivo turístico busca fortalecer la oferta e infraestructura de servicios turísticos como una actividad estratégica para impulsar el crecimiento económico del Municipio. Al promover el turismo como un sector fundamental para potenciar el desarrollo, se busca atraer inversiones y generar empleos permanentes y bien remunerados, además se plantea una oferta turística integradora y sustentable que atienda las necesidades del lugar. Se destaca principalmente el ecoturismo, el turismo de aventura, recreación y esparcimiento, el turismo de negocios y el de fomento cultural [42].

Al utilizar la Teoría General de Sistemas (TGS), se cuestiona la naturaleza del sistema que se estudia, así como también su contexto dentro de un supra sistema, con el fin de focalizar cuál es su propósito. Bajo la TGS se investigan conceptos y métodos sistémicos, dicho enfoque está encaminado a estudiar fenómenos diseñados y estructurados por el hombre, lo que conlleva describir las relaciones generales del sistema. Se puede desarrollar una nueva forma de pensamiento sistémico y científico de aproximación a la realidad, con una orientación hacia prácticas transdisciplinarias dentro del paradigma de la TGS. Este incorpora una perspectiva holística e integradora, donde las relaciones juegan un papel muy importante, y donde el objetivo principal es localizar similitudes entre estructuras y propiedades de los fenómenos que se llevan a cabo dentro del mismo.

Algunos autores desde su perspectiva científica y sus valores filosóficos han construido una visión del mundo donde el pensamiento de sistemas organiza de manera más clara la complejidad del mundo real. Al mismo tiempo, han buscado la solución de problemas basados en cualquiera de los sistemas que se pueden trabajar. La Metodología de Sistemas Suaves (SSM) de Checkland [43] considera la incorporación del entorno y el nivel cultural de los sistemas involucrados que contribuyen a determinar el sistema principal y su subsistema, así mismo, los elementos que se ven envueltos en el proceso principal. Está basada en interpretaciones que permiten comprender y dar solución a problemas; posteriormente se determina un esquema para la toma de decisiones.

De acuerdo con dicha metodología, se determina el cálculo de la muestra considerando a los visitantes, con un nivel de confianza de $95 \%$ y un porcentaje de error del $5 \%$ en una población de 350 visitantes sin influencia de temporalidad. La información que se obtuvo muestra las características generales de los turistas que visitan el municipio de Villa de Tezontepec, su gasto promedio por estancia y, finalmente, la percepción que tienen sobre el municipio; para ello se aplica- 
ron 183 encuestas a turistas por medio de un muestreo aleatorio simple.

El objetivo de recopilar estos datos es conocer cómo se integran las MIPYMES gastronómicas y sus alcances dentro del municipio de Villa de Tezontepec (véase Fig. 2), debido a que este tipo de empresas y el turismo gastronómico constituyen un subsistema que se incluye dentro de otro sistema aún más amplio que es la sociedad con la que interactúan. Este complejo más grande está integrado por grupos de trabajo que responden a una determinada estructura y se ven inmersos en un contexto de turismo y desarrollo local, por tal motivo, se deben conocer los subsistemas que forman de manera general dicho sistema de integración más amplio: MIPYMES gastronómicas.

Las relaciones de los sistemas de estudio son múltiples. Cada uno tiene una serie de conexiones que a su vez influyen de manera directa en otro sistema. Para determinar el sistema de desarrollo local se necesita analizar los alcances y límites de cada elemento, los cuales darán a conocer cómo es su desempeño, sus interrelaciones y resultados, para establecer si la generación del desarrollo local es resultado de su desempeño en el municipio y genera cambios notables en la calidad de vida de los habitantes que se encuentran inmersos en el sistema de estudio.

Considerar a toda la población del municipio sería la manera más exacta de acercarse al objeto de estudio, es una labor compleja y exhaustiva en la práctica; entre los motivos principales que lo impiden se encuentran la escasez de recursos

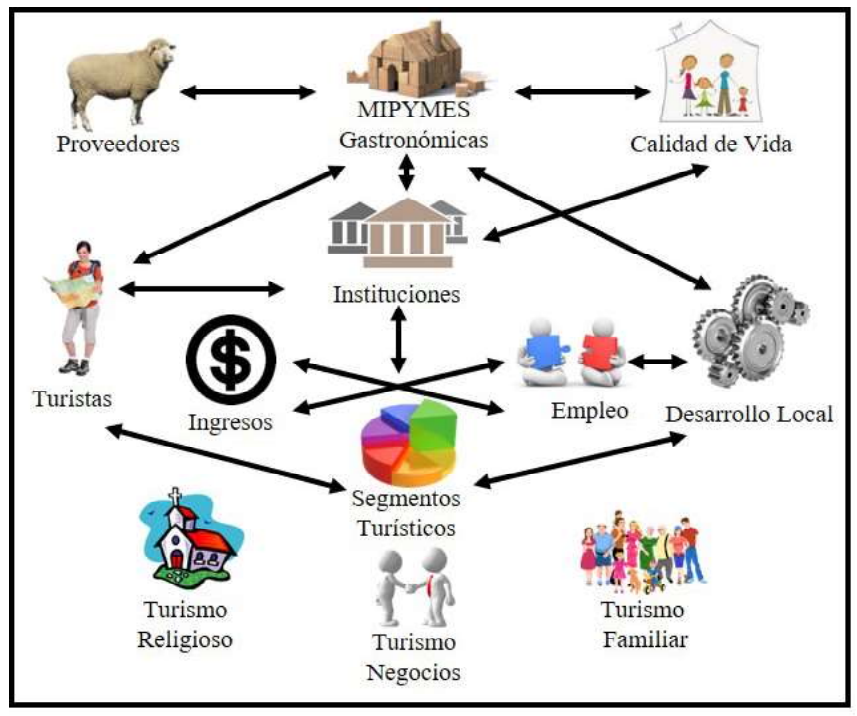

Fig. 2. Apreciación de los sistemas de estudio. humanos y económicos, y la dificultad para acceder a todos los sujetos. Es por esta razón que se estudia solo a una parte de los habitantes; posteriormente, se hace una generalización de los resultados obtenidos y a partir de ellos se infieren los datos que podrían abarcar a toda la población. Por ello, fue necesario, en primera instancia, determinar el tamaño de la muestra para nuestro trabajo de investigación.

Dentro del análisis de los datos se muestra una visión enriquecida, con la finalidad de obtener posibles sistemas relevantes, su entorno y sus interrelaciones. Con la ayuda de cuadros se determinan los elementos que actúan dentro del sistema de desarrollo local y donde se encuentran los siguientes sistemas de estudio:

- El visitante es la persona que permanece menos de 24 horas en el lugar al que viaja.

- Las MIPYMES gastronómicas son empresas que se dedican al giro gastronómico, esencialmente a la elaboración de Barbacoa de borrego de manera artesanal.

- Dentro de las instituciones el municipio cuenta con una Dirección de Turismo, que, dentro del plan municipal de desarrollo, considera al turismo como una de las principales actividades económicas.

- El desarrollo local es el resultado del estudio de los sistemas mencionados anteriormente: sus alcances, beneficios, cambios positivos y negativos que ha tenido el municipio.

Lo que se observa en primer lugar, es que los visitantes buscan una alternativa gastronómica dentro del estado de Hidalgo; ahí las MIPYMES gastronómicas destacan entre otras empresas; mientras tanto, las instituciones regulan la actividad turística del municipio, con reglamentos, cursos y apoyos económicos para que el turismo en el municipio se pueda colocar como una de las principales actividades económicas a nivel estatal. Finalmente, dentro de este sistema, el desarrollo local se observa como resultado; desde hace tiempo, la actividad turística derivada de la gastronomía, ha colocado al municipio como uno de los primeros dentro de este sector, principalmente por la barbacoa de borrego: los visitantes, al momento de realizar su visita con motivo de comer barbacoa, generan un flujo económico que beneficia y tiene un impacto sobre las MIPYMES en general, es decir, que también se benefician otros rubros como las artesanías, la recreación y demás alimentos. Por su parte, el entorno nos muestra los agentes que influyen de manera directa en el desarrollo local.

\section{Resultados}

El uso de indicadores ha sido y sigue siendo una práctica muy habitual en la gestión de destinos turísticos, pues proporciona un conjunto de señales, regulaciones y datos para 
la observación, entre otras cosas, de políticas y decisiones gubernamentales, a pesar de la dificultad implícita que tiene el sector turístico [44].

En la figura 3 muestra los datos recolectados y puede considerarse como uno de los principales indicadores del desempeño turístico del municipio de Villa de Tezontepec. La información muestra que el $47 \%$ de los visitantes que viajan al municipio por motivos gastronómicos, debido a la creciente demanda del mercado potencial de este tipo de turismo y también debido su localización como punto de acceso al estado de Hidalgo. En cambio, el turismo religioso no recibió ninguna apreciación por parte de los turistas, mientras que el $20 \%$ de los visitantes solo viaja al municipio por motivos de recreación; por otra parte, el $26 \%$ lo considera dentro de su elección por brindar un entorno familiar y al $7 \%$ restante le agrada realizar compras de productos que se elaboran en el municipio (talabartería).

\section{MIPYMES del municipio de Villa de Tezontepec}

El municipio cuenta con 688 unidades económicas de las cuales 89 se dedican a la prestación de servicios de alimentos y bebidas y, entre ellas, solo 6 establecimientos de venta de barbacoa de borrego han sido registrados ante el INEGI. Cabe señalar que, en su mayoría, las MIPYMES gastronómicas no se encuentran dentro del registro como unidades económicas, debido a que están en calidad de comercio ambulante.

Con tan solo el $12 \%$ de unidades económicas en el municipio, se recibe a una cantidad considerable de visitantes que realizan turismo gastronómico (véase Fig. 4), lo que favorece a la comunidad local con mayores ingresos, mismos que benefician su imagen y el contribuyen a su reconocimiento. Por otro lado, la localización de los comercios fijos y ambulantes genera una oferta diversa.

\section{Gasto promedio}

La edad media de los turistas que visitan el municipio fue de 34 años, mientras que la moda se mantuvo en los 40 años. En su mayoría, las personas que viajan son familias que buscan

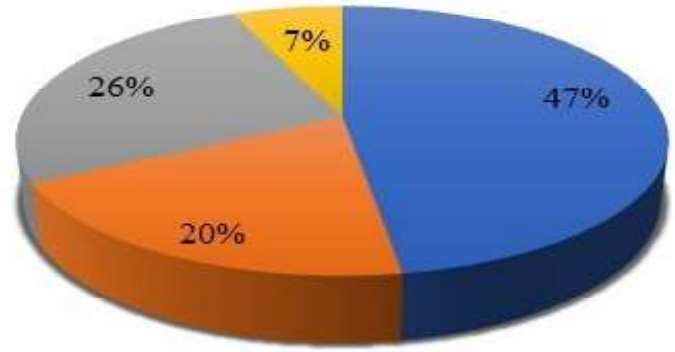

Fig. 3. Motivo de Visita al Municipio de Villa de Tezontepec.

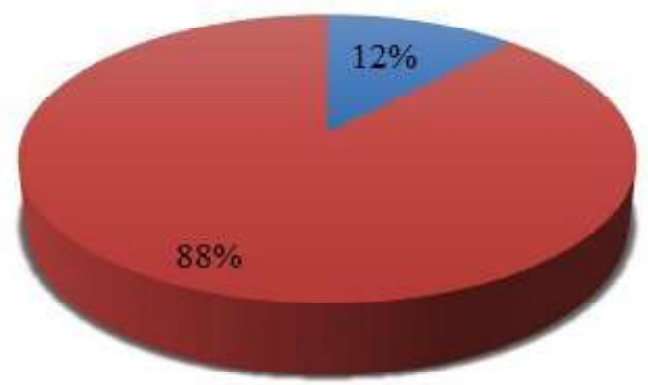

Fig. 4. MIPYMES gastronómicas.

un ambiente agradable y realizan su visita en grupo (familia). De los visitantes encuestados, el $57 \%$ son hombres y el $43 \%$ son mujeres, como consecuencia el mercado gastronómico es el más beneficiado. El gasto promedio de los visitantes en su estadía juega un papel muy importante para el desarrollo personal, familiar y local del municipio pues el $47 \%$ de los visitantes realiza su visita debido al aspecto gastronómico, lo que distribuye el gasto en su mayoría por concepto de la barbacoa de borrego.

E1 54\% de los visitantes realizó un gasto promedio de \$200 a $\$ 600$ por familia, por consumo de alimentos. Si se considera que el precio por kilo de barbacoa de borrego en promedio es de $\$ 350$, la derrama por concepto del consumo de este alimento genera para las MIPYMES un ingreso potencial para activar la economía municipal, ya que el gasto medio de los visitantes oscila entre los $\$ 600$ y $\$ 1000$, y poco más del $15 \%$ gasta entre $\$ 1000$ y $\$ 2000$ a lo largo de su permanencia en el municipio (véase Fig. 5).

\section{Análisis relación costo beneficio}

La barbacoa de borrego es un platillo tradicional del estado de Hidalgo, pero la particularidad que tiene la preparación en el municipio de Villa de Tezontepec es que se hornea bajo tierra. Dicha peculiaridad constituye la fuente motivadora del desplazamiento de los visitantes. Debido a ello el, estudio se centró en las MIPYMES gastronómicas que se dedican a la elaboración y venta de barbacoa, lo que ha fomentado el incremento de visitas que benefician a la comunidad en general.

Los beneficios de algo deben ser correspondientes a lo que las personas están dispuestas a pagar por eso, resaltando las condiciones sobre la distribución del ingreso y la disponibilidad de la información, mientras que un análisis de costo beneficio consiste en cuatro pasos principales: 1. identificación de los costos y beneficios relevantes; 2. Medición de los costos y de los beneficios; 3. Comparación de los flujos Las unidades económicas que se desempeñan dentro del 


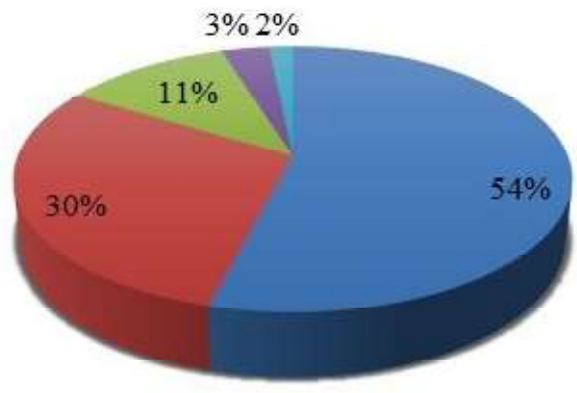

200 a 600

600 a 1000

1000 a 1500

1500 a 2000

Más de 2000

Fig. 5. Gasto promedio por día de permanencia en el municipio.

municipio generan ingresos al municipio por concepto de pago de piso, pago de alta y pago mensual de actividades (asociación). Estas unidades generan una cadena de valor que se inicia con la visita de turistas al municipio, donde las MIPYMES captan la demanda y, a su vez, retribuyen al municipio con un pago. Los costos de producción (véase Fig. 6) por concepto de la elaboración de barbacoa (un borrego) determinan la ganancia que cada productor percibe en un día laboral; cada productor prepara al menos tres borregos por día de venta, con un porcentaje de beneficio del $50 \%$, y obtiene un total de $\$ 2,065.75$ como costo total de producción por cada borrego con un peso de 30 kilos aproximadamente.

En la preparación de barbacoa, cada MIPYME tiene como merma el 50\% de cada borrego. Dicha merma consta de huesos, vísceras y piel, que pueden vender a otras MIPYMES que se dedican a la talabartería. A partir de la ganancia que se tiene de cada borrego aún se deben considerar los sueldos y otros gastos. Principalmente la familia es quien otorga el servicio a los turistas, con ayuda de algunos empleados (cargadores), así el egreso es menor. Si se considera el costo por

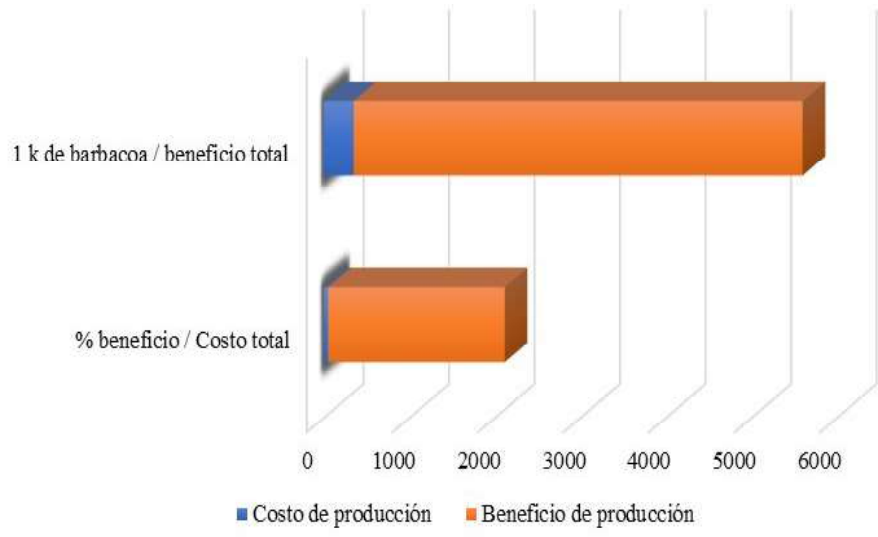

Fig. 6. Costo beneficio de la producción. kilo de barbacoa en $\$ 350.00$ y 15 kilos por cada borrego (3 borregos por 3 días de venta), se obtiene el beneficio total mensual de $\$ 16,364.6$ por45 kilos de barbacoa, ofertados de sábado a lunes con una utilidad de $\$ 4,091.15$.

De las 18 MIPYMES contabilizadas en la actividad gastronómica que proviene de la venta de Barbacoa, solo 12 cuentan con las características que se contemplan dentro de este análisis. Por otro lado, tan solo 6 MIPYMES consideran gastos que les permiten promover su negocio para atraer clientes y así obtener mayores ingresos. Estas seis MIPYMES se encuentran registradas dentro del Directorio Estadístico Nacional de Unidades Económicas (DENUE) y obtienen un beneficio total por $\$ 16,364.6$ pesos mensuales en 12 días laborables, lo que demuestra que los beneficios derivados del consumo de barbacoa de borrego son considerables.

Los beneficios que aportan las MIPYMES de barbacoa de borrego al turismo gastronómico y al municipio de Villa de Tezontepec son positivas. Pero el municipio no cuenta con la regulación potencial para estos productores, pues, si se estima que los beneficios son para las familias que se desempeñan dentro del mismo giro comercial, las condiciones de generación de empleos son reducidas, lo que determina la distribución de los ingresos exclusivamente para estos vendedores. El gasto tiene una distribución limitada: por 3 días laborables las MIPYMES realizan una derrama mensual al municipio de $\$ 7,048.8$ ( $\$ 84,585.6$ pesos anuales de las 18 unidades económicas), ingresos que pueden ser empleados para la mejora de áreas verdes, estructura (parques, mercado, servicios al turista) e infraestructura (carreteras, telecomunicaciones, señalamientos) que los turistas utilizan para llegar al municipio.

Podríamos observar mayores beneficios del sistema del turismo gastronómico si se tuviera la posibilidad de regularizar el resto de las MIPYMES, pues de este modo, se beneficiarían otras familias, lo que, al mismo tiempo, haría que la cadena de valor incrementara para bien de la localidad, de la misma forma que para los prestadores de servicios turísticos complementarios. Los beneficios que aporta el turismo gastronómico al desarrollo local del municipio son vastos, gracias a ellos se activa la economía e incrementa la llegada de turistas. Por esta razón, el municipio se coloca como un destino gastronómico importante dentro del estado de Hidalgo desde hace ya varios años. De esta forma, se demuestra que la actividad turística trae consigo beneficios endógenos para la localidad, que se ven reflejados en el desarrollo de productos y servi- 
cios a mediano plazo. Es así como el presente proyecto de investigación determinó un sistema de desarrollo local analizando sus relaciones (procesos de entradas y salidas).

\section{Conclusiones}

En el presente trabajo de investigación se analizó el desempeño de la actividad turística gastronómica en el municipio de Villa de Tezontepec, así como los alcances que se presentan en el desarrollo de la localidad. El municipio ha sobresalido dentro del estado de Hidalgo por su oferta gastronómica, cuyo platillo principal es la barbacoa de borrego.

Para poder determinar el sistema de estudio se llevó a cabo una investigación que permitió conocer más a fondo la actividad turística, misma que genera cambios dentro del municipio al ser una de las actividades principales. El sistema turístico dentro del municipio no presentaba, de manera concreta, una estructura funcional donde se logrará descubrir qué importancia tiene el turismo gastronómico para los pobladores, pues las MIPYMES turísticas gastronómicas fungen como el principal impulsor y motor de la actividad.

El sistema está integrado por visitantes, conceptualizados como excursionistas que permanecen en el lugar que visitan no más de 24 horas. Dentro del sistema, representan las entradas que detonan la actividad turística $y$, en promedio, gastan de 600 a 1200, y se constituyen en familias de 4 a 6 integrantes.

El segundo integrante son las MIPYMES gastronómicas, específicamente las que se dedican a la venta de barbacoa de borrego; como prestadores de servicios turísticos, generan un sistema que atrae a los visitantes por medio de su oferta, así mismo, fungen como uno de los principales actores de la actividad económica municipal, al crear empleos. Estas MIPYMES obtienen beneficios económicos importantes; sus ganancias se estiman a partir de las instituciones que regulan la actividad turística del municipio. Por su parte, la dirección de turismo del municipio apoya a las MIPYMES para su establecimiento y promoción, del mismo modo que lo hace con el resto de los atractivos, todo ello genera una oferta integradora de servicios $\mathrm{y}$ actividades para una mayor captación de visitantes.

El sistema que se obtuvo (véase Fig. 7), demuestra la integración de las MIPYMES turísticas gastronómicas con los visitantes, quienes activan la economía local, cuyos alcances impactan de forma positiva no sólo en la gastronomía sino también en los productores locales (artesanías de piel: curtidoras dentro del sistema de las MIPYMES, que son quienes comercializan la materia prima), vendedores ambulantes (internos y externos), transporte público, otros prestadores de servicios de alimentos y bebidas. Dentro de este sistema, el

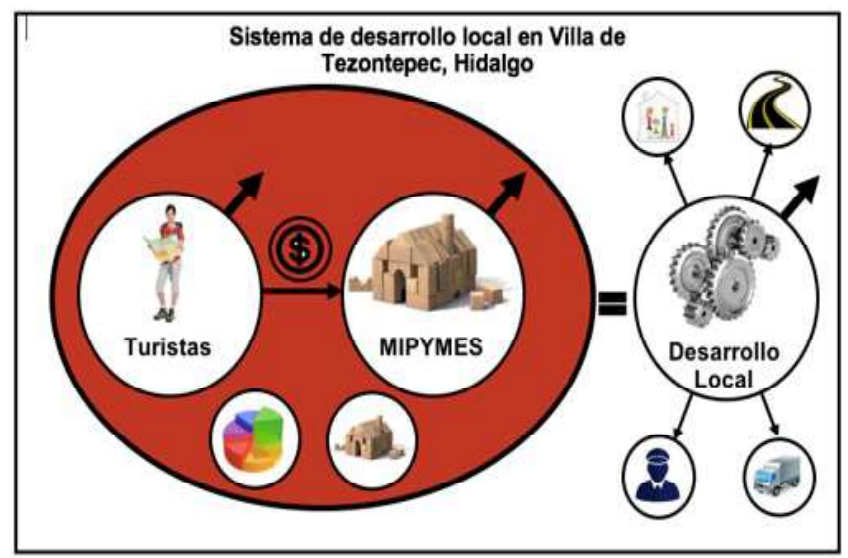

Fig. 7. Sistema de desarrollo local del municipio de Villa de Tezontepec, Hidalgo.

municipio gestiona apoyos para la mejora de infraestructura y brinda capacitación y gestión para la apertura de nuevos centros turísticos con la finalidad de mantener una estructura demandante para los visitantes.

La comunidad local inmersa en el sistema turístico ha logrado una mejora tanto en su calidad de vida como en su desarrollo personal y laboral, pues la generación de empleo no solo se limita al ámbito familiar si no que tiene alcance local, lo que beneficia en cadena a muchas familias del municipio de Villa de Tezontepec. Gracias a la actividad turística gastronómica, el desarrollo local ha generado grandes beneficios para los pobladores; al activar la económica local, los beneficios que aporta el turismo tienen amplio alcance.

La actividad turística ha demostrado cambios positivos dentro del sistema, gracias al desempeño de las MIPYMES gastronómicas en el municipio de Villa de Tezontepec. Estos se generan a partir del desplazamiento de personas al municipio con fines gastronómicos; pero este desplazamiento influye también en otras actividades que no son gastronómicas. Con el aprovechamiento adecuado de los recursos, el municipio podrá determinar si los impactos del turismo han aportado cambios de manera positiva para el municipio; los aportes de las MIPYMES gastronómicas ayudarán a conseguir el objetivo que persigue el municipio: elevar su importancia turística. Se establece la respuesta global del problema, son los objetivos alcanzados, las hipótesis comprobadas, modificadas o rechazadas.

\section{Agradecimientos}

Con especial agradecimiento al doctor Juan Bory Reyes por su interés y aportes a este trabajo de investigación, a la sección de investigación y posgrado por las facilidades. 


\section{Referencias}

[1] Organización Mundial de Turismo, Recomendaciones Internacionales para Estadísticas de Turismo, 2010. Disponible en: http://unstats.un.org/unsd/publication/ SeriesM/Seriesm_83rev1s.pdf

[2] L. Coriolano, "Os limites do desenvolvimento e do turismo,". PASOS: Revista de Turismo y Patrimonio Cultural, vol. 1 no. 2,pp. 161-171, 2003.

[3] Secretaría de Turismo, Convenio SECTUR-FONATURNAFINSA para Financiamiento a Empresas turísticas, México: SECTUR, 2015.

[4] Consejo de Promoción Turística de México y Sistema Integral de Información de Mercados Turísticos, Situación del sector turístico 2013 y Avances, 2014.

[5] Organización para la Cooperación y el Desarrollo Económico, Estudio de la Política Turística de México, 2017. Disponible en: http://dx.doi.org/10.1787/9789264266575-en

[6] C. Varisco, "Sistema productivo turístico y desarrollo local," Ciencias Sociales Online, vol. 2, no. 4, pp. 15-36, 2007.

[7] Instituto Latinoamericano y del Caribe de Planificación Económica y Social, Manual de desarrollo local, pp. 1-201, 1998.

[8] S. Martínez, L. Flamand, y A. Hernández, "Panorama del desarrollo municipal en México. Antecedentes, diseño y hallazgos del índice de desarrollo municipal básico," Gestión y Política Pública, vol. 17, no.1, pp. 145-192, 2008.

[9] J. Ivars, Planificación y gestión del desarrollo turístico sostenible: propuesta para la creación de un sistema de indicadores, Comisión Interministerial de Ciencia y Tecnología, vol. 1, pp.1-6, 2001.

[10] A. Vázquez-Barquero, Desarrollo local, una estrategia para tiempos de crisis, conceptos críticos, Universitas Forum, vol. 1, no. 2, pp. 1-11, 2009.

[11] B. Vachon, El Desarrollo Local. Teoría y Práctica. Reintroducir lo Humano en la Lógica de Desarrollo, España: Trea, 2001.

[12] S. Boisier, "Desarrollo (local): ¿De qué estamos hablando? Transformaciones globales, instituciones y políticas de desarrollo local," Homo Sapiens, pp.48 -74, 2001.

[13] P. Di Pietro, Hacia un desarrollo integrador y equitativo: una introducción al desarrollo local, 2014. Disponible en: http://www.iepala.es/IMG/pdf/Articulo_Di_Pietro_ Desarrollo_Local_2_.pdfConsultado: 27 julio, 2015.

[14] F. Alburquerque, Metodología para el desarrollo económico local, Instituto Latinoamericano y del Caribe de Planificación Económica y Social (ILPES), Comisión Económica para América Latina y el Caribe (CEPAL), 1997.

[15] M. Cienfuegos, A. Aguilar, "Actores/Agentes universitarios para el desarrollo local," Espacios Públicos, pp. 216-226, 2011.
[16] J. Mantero, "Desarrollo local y actividad turística," Aportes y Transferencias, vol. 1, no. 8, pp. 11-382004.

[17] P. Costamagna, Iniciativa de desarrollo económico local. La articulación y las interacciones entre instituciones. El caso de Rafaela. Santiago de Chile, CEPAL, 1999.

[18] E. Lozano, J. M. Ruíz, M. D. Riveroll, "Las Micro, Pequeñas y Medianas Empresas como las grandes generadoras de empleo en México," CROSS, no. 8, pp. 1-23, 2013.

[19] A. De la Rosa, "La Micro, Pequeña y Mediana Empresa en México: sus saberes, mitos y problemática," Iztapalapa, no. 48,pp. 183-220, 2000.

[20] Cuenta Satélite de Turismo de México, Glosario; Turismo, 2012.

[21] Plan Nacional de Desarrollo, Turismo México, México: SEGOB, pp. 656, 2012.

[22] Instituto Nacional de Estadística y Geografía, Micro, Pequeña, Mediana y Gran Empresa, estratificación de los establecimientos, México: Censos Económicos 2009.

[23] Consejo de Promoción Turística de México, Panorama del sector turístico, 2015.

[24] Instituto Nacional de Estadística y Geografía, Directorio Estadístico Nacional de Unidades Económicas - DENUE, México: INEGI, 2015.

[25] Instituto Nacional de Estadística y Geografía, México: Conociendo a México, México: INEGI, 2012.

[26] Secretaría de Gobernación, Sexto Informe de Gobierno, Economía Competitiva y Generadora de Empleos, México: SEGOB, 2012.

[27] El Universal. MIPYMES en México, enero 2011. Disponible en: http://www.discoveryamericas.com/periodicos/ MPyMEs\%20en\%20Mexico\%20-Enero\% 202011.pdf

[28] PROMEXICO, PYMES, Eslabón Fundamental para el Crecimiento en México, 2015. Disponible en: http:// www.promexico.gob.mx/negocios-internacionales/pymeseslabon-fundamental-para-el-crecimiento-en-mexico.html

[29] M. Mejía, "Integración, gestión y capacitación del recurso humano en las MIPYMES turísticas en la ciudad de Guanajuato," El Periplo Sustentable, no. 32, febrero 2017. Disponible en: https://rperiplo.uaemex.mx/article/ view/4875.

[30] Secretaría de Gobernación, Quinto Informe de Labores 20162017 SECTUR, México: SEGOB, 2017.

[31] Secretaría de Turismo, "México inicia actividades del año dual con lo más destacado de su gastronomía contemporánea," México: SECTUR, 2015.

[32] Secretaría de Turismo, Rutas gastronómicas, México: SECTUR, 2012. Disponible en: http://rutasgastronomicas. sectur.gob.mx/descargas/guia_rapida.pdf

[33] Secretaría de Turismo, Reconocerán a la gastronomía mexicana en el 50 best restaurants of the world en Londres, México: SECTUR, 2015. 
[34] Organización para la Cooperación y el Desarrollo Económico, Tendencias y políticas del turismo de la OCDE 2012, pp. 1-6, 2012.

[35] G. Millán, E. Morales, L. Pérez, "Turismo gastronómico, denominaciones de origen y desarrollo rural en andalucía: situación actual," Boletín de la Asociación de Geógrafos Españoles, no. 65, pp. 113-137, 2014. Disponible en: 10.21138/bage. 1746

[36] N. Bringas, J. González, "El turismo alternativo: una opción para el desarrollo local en dos comunidades indígenas de Baja California," Economía, Sociedad y Territorio, vol. 4, no.15, pp. 551-558, 2004.

[37] R. García, J. Andrés y C. Espejo, "Conocimientos tácticos, transversalidad turística y desarrollo local. El ejemplo de una ciudad desolada y en proceso de reconvención: Lorca (Región de Murcia, España)," PASOS: Revista de Turismo y Patrimonio Cultural, vol.14 no. 5, pp. 1093-1107, 2016.
[38] B. Barbini, G. Cruz, N. Roldán, M. Cacciutto, "Modelos de desarrollo e implicancias en el turismo: un análisis histórico," Registros, vol. 8 no. 9, pp. 117-129, 2012.

[39] Secretaría de Desarrollo Económico - Hidalgo, Hidalgo en cifras. México. México: SEDECO-Hidalgo, 2015. Disponible en: http://sedeco.hidalgo.gob.mx

[40] Programa Sectorial de la Secretaría de Turismo y Cultura, Sectores económicos del estado de Hidalgo, 2012.

[41] Villa de Tezontepec, Página web oficial, México. Municipio de Villa de Tezontepec, 2015. Disponible en: http://villadetezontepec.gob.mx/

[42] Plan Municipal de Desarrollo, Villa de Tezontepec, Hidalgo, México, 2012.

[43] P. Checkland, Pensamiento de sistemas. practica de sistemas. $7^{\mathrm{a}}$ ed., México: Limusa, 2005.

[44] E. Torres, "¿Qué indica un indicador? Análisis Comparativo de los Destinos Turísticos," Revista de Análisis Turístico, vol. 2, no.2,pp.69-87, 2006. 
